\title{
Predictors of failure following fixation of intertrochanteric fractures with proximal femoral nail antirotation
}

\author{
Raghavan Raghuraman ${ }^{1}$, MBBS, MMed, Jia Wen $\underline{K a m}^{2}$, MSc, David Thai Chong Chua ${ }^{1}$, MBBS, FRCSEd
}

\begin{abstract}
INTRODUCTION This retrospective matched case-control study aimed to identify predictors of cut-out following intramedullary nailing of intertrochanteric fractures with the 200-mm Synthes proximal femoral nail antirotation (PFNA). METHODS 609 patients underwent intramedullary nailing for intertrochanteric fractures at our institution between January 2011 and December 2014. 370 patients satisfied the inclusion criteria. There were 20 cases of implant cut-out. Cases and controls were matched using a propensity score-matching method with an $\mathrm{m}: \mathrm{n}$ ratio, matching the criteria of gender, age and side of operation. Radiographs were assessed to determine fracture classification, fracture reduction quality, tip-apex distance, calcar referenced tip-apex distance (CalTAD), anteroposterior (AP) Parker's ratio index, lateral Parker's ratio index and cervical angle difference. Conditional logistic regression analysis was performed to determine any association between potential predictors and cut-outs.

RESULTS The cut-out incidence was $5.4 \%$. Of the 20 cut-outs, 16 were superior and four were cut-throughs. Univariate analysis only showed a significant association between unsatisfactory fracture reduction quality and cut-outs (odds ratio [OR] 10.1, 95\% confidence interval $[\mathrm{Cl}] 1.31-77.6, p=0.027)$. This association remained significant with multivariate logistic regression analysis (OR $16.4,95 \% \mathrm{Cl} 1.9-140.4, \mathrm{p}=0.011$ ). Cut-throughs had significantly lower CalTAD (16.2 vs. $27.5, p=0.016)$ and AP Parker's ratio index values (38.7 vs. $50.7, p=0.007)$ than superior cut-outs.

CONCLUSION Unsatisfactory fracture reduction quality was a significant predictor of cut-out in intertrochanteric fractures treated with the 200-mm PFNA. Cut-outs had two distinct modes, with cut-throughs having a deeper and more inferior helical blade position in the femoral head compared to superior cut-outs.
\end{abstract}

Keywords: cut-out, failure, intertrochanteric fracture, intramedullary nailing, predictors

\section{INTRODUCTION}

The incidence of hip fractures has seen a rising trend in recent times and is expected to continue increasing as the population ages. ${ }^{(1,2)}$ Hip fractures are associated with considerable morbidity as well as mortality for patients. ${ }^{(3)}$ They also present a considerable economic burden to society. ${ }^{(4,5)}$ This cost is further increased in the event of failure of treatment in this group of patients. ${ }^{(5)}$ Hence, it is pertinent to prevent treatment failures whenever possible.

The most common cause of failure of fixation of intertrochanteric fractures is lag screw cut-out. ${ }^{(6,7)}$ Predictors of lag screw cut-out have been extensively studied and described in the literature, mostly involving the fixation of intertrochanteric fractures with the dynamic hip screw (DHS). ${ }^{(6-10)}$ Recommendations on the ideal lag screw position within the femoral head, including the tip-apex distance (TAD) rule $(<25 \mathrm{~mm})$, are based on studies involving fixation using the DHS. ${ }^{(7,11)}$ These recommendations may not be applicable to fixation of intertrochanteric fractures using intramedullary devices, such as the Synthes proximal femoral nail antirotation (PFNA; Synthes, Oberdorf, Switzerland), in view of the inherent differences in implant design and biomechanical properties. However, there is a relative scarcity of literature on the predictive factors for implant cut-outs in intramedullary nailing of intertrochanteric fractures. This retrospective matched casecontrol study aims to determine the incidence of cut-outs among intertrochanteric fractures treated with the 200-mm PFNA, identify if fracture type is a predictor of cut-out and identify any technical factors, including quality of fracture reduction and implant position, that may influence the development of cut-outs.

\section{METHODS}

All patients who were treated with intramedullary nailing for an intertrochanteric fracture at Changi General Hospital, Singapore, between January 2011 and December 2014 were retrospectively reviewed. All cases were reviewed for gender, side of fracture, age at time of surgical fixation, date of final clinical follow-up and type of implant used. Patients who had sustained a non-osteoporotic pathological fracture, had less than 90 days of radiological followup and had undergone fixation with any implant other than a 200-mm PFNA were excluded from the study.

All postoperative radiographs of patients who met the inclusion criteria were reviewed to identify implant cut-outs. 'Cutout' was defined as penetration of the implant through the femoral neck or head, as observed on the radiographs. For each case of a cut-out, it was also noted whether the cut-out occurred superiorly with varus collapse of the fracture or whether it occurred as a medial cut-through of the blade through the femoral head. The remaining patients who met the inclusion criteria and had not experienced a cut-out were used as a pool for controls. Cases and controls were matched using a propensity score-matching

${ }^{1}$ Department of Orthopaedic Surgery, ${ }^{2}$ Clinical Trials and Research Unit, Changi General Hospital, Singapore

Correspondence: Dr Raghuraman Raghavan, Senior Resident, Department of Orthopaedic Surgery, Changi General Hospital, 2 Simei Street 3 , Singapore 529889. equinox2309@gmail.com 

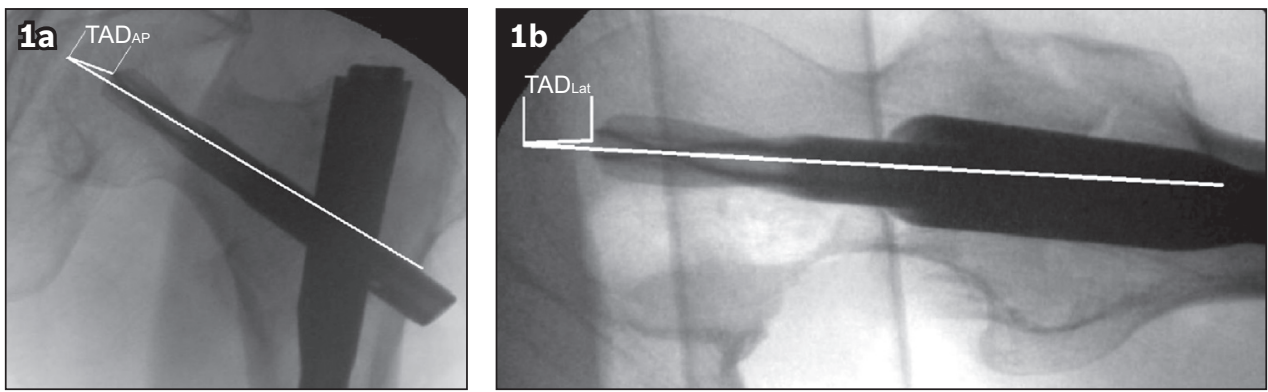

Fig. 1 Radiographs show measurement of the (a) the anteroposterior component $\left(T A D_{A P}\right)$ and (b) the lateral component (TAD $\left.{ }_{\text {Lat }}\right)$ of the $T A D$. The apex of the femoral head was determined by using a guideline running through the centre of the femoral head and neck. TAD: tip-apex distance

method with an m:n ratio, matching the criteria of gender, age at time of operation and side of operation.

Preoperative radiographs were reviewed to classify the fractures based on the Orthopaedic Trauma Association (AO/OTA) classification system. Fractures were subdivided into classes 31.A1, 31.A2 and 31.A3 accordingly. Intraoperative fluoroscopy images were used to assess the TAD, calcar referenced tip-apex distance (CalTAD), anteroposterior (AP) Parker's ratio index, lateral Parker's ratio index and fracture reduction quality. Measurements were corrected for magnification by using the known diameter of the PFNA helical blade as reference for both the AP and lateral views. The TAD was defined as the sum of the distance, in millimetres, from the tip of the helical blade to the apex of the femoral head, as measured on the AP radiograph and lateral radiograph, as described by Baumgaertner et al ${ }^{(7)}$ (Fig. 1). The AP measurement for CalTAD (CalTAD ${ }_{A \mathrm{AP}}$ ) was obtained by first identifying the point of intersection between the femoral head and a line drawn just adjacent to the medial cortex of the femoral neck and parallel to a guideline drawn through the centre of the femoral head and neck (Fig. 2). CalTAD ${ }_{\mathrm{AP}}$ measured in millimetres, was then added to the lateral component of the TAD to obtain CalTAD as described by Kuzyk et al. ${ }^{(12)}$ The AP and lateral positions of the helical blade with respect to the femoral head were measured using the AP and lateral Parker's ratio index, respectively. ${ }^{(9)}$ Values derived using this method would fall within a range of $0-100$, with lower values representing a more inferior and posterior position within the femoral head.

Quality of fracture reduction was categorised as either satisfactory or unsatisfactory based on a modification of the method described by Baumgaertner et al. ${ }^{(7)}$ Reduction quality was evaluated on the basis of displacement and angulation criteria. The displacement criterion was met if there was less than $4 \mathrm{~mm}$ of displacement of the fracture fragments on the AP and lateral radiographs. The angulation criterion was met if the neck shaft angle was normal or slightly valgus $\left(130^{\circ}-150^{\circ}\right)$ and there was less than $20^{\circ}$ of angulation on the lateral radiograph. Quality of fracture reduction was categorised as satisfactory if both criteria were met and unsatisfactory if either was not met. Cervical angle difference was calculated by measuring the difference in the femoral neck-shaft angles of the operated and non-operated sides in the immediate postoperative AP pelvic radiograph. Positive cervical angle difference values represented a more valgus alignment of the operated sign as compared to the non-operated

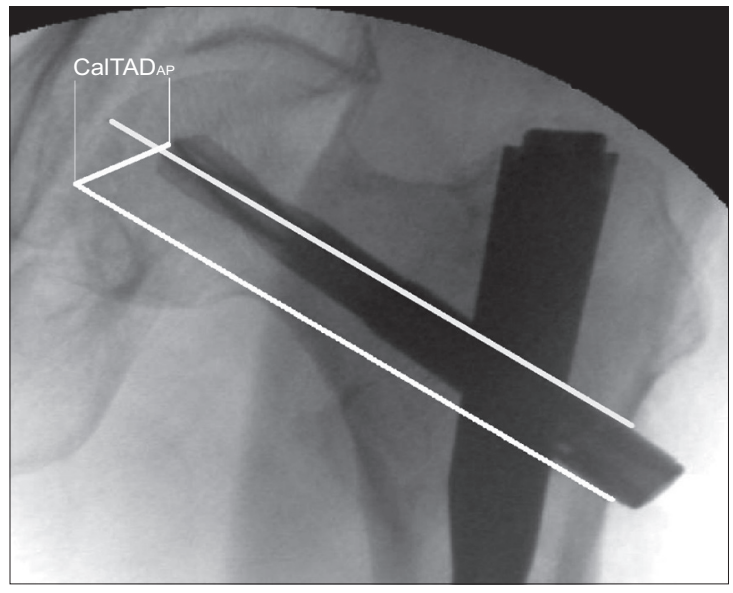

Fig. 2 Radiograph shows measurement of the anteroposterior (AP) component of the calcar referenced tip-apex distance $\left(C a l T A D_{A P}\right)$. The guideline used to determine the apex of the femoral head was moved inferiorly such that it runs parallel to the original guideline and lies adjacent to the medial cortex of the femoral neck.

side, while negative values represented a more varus alignment on the operated side compared to the non-operated side.

Categorical variables were presented as percentages. Numeric variables were presented as mean \pm standard deviation for parametric distributions and median (interquartile range) for non-parametric distributions. Differences in characteristics were examined using chi-square test or Fisher's exact test for categorical variables, and two-sample $t$-test or Mann-Whitney $U$ test for continuous variables, where appropriate.

Cut-outs (cases) and non-cut-outs (controls) were matched by age at time of operation, gender and side of operation. Conditional logistic regression analysis was performed to determine the association between potential predictors and development of cut-outs. Odds ratios (OR) associated with their respective 95\% confidence intervals $(\mathrm{Cl})$ were presented. A two-tailed p-value $<0.05$ was considered statistically significant. Statistical analysis was performed using IBM SPSS Statistics version 19.0 (IBM Corp, Armonk, NY, USA).

\section{RESULTS}

A total of 609 intertrochanteric fractures underwent intramedullary nailing at our institution between January 2011 and December 2014. Of these, 97 patients underwent intramedullary nailing with either long nails or implants other than the 200-mm Synthes PFNA, hence were excluded from the study. 140 cases did not 
Table I. Univariate analysis of predictors for cut-outs.

\begin{tabular}{|c|c|c|c|c|c|}
\hline \multirow[t]{2}{*}{ Parameter } & \multicolumn{3}{|c|}{ No. $(\%) /$ mean \pm SD } & \multirow[t]{2}{*}{ OR (95\% CI) } & \multirow[t]{2}{*}{ p-value } \\
\hline & All $(n=86)$ & Non-cut-out $(n=66)$ & Cut-out $(n=20)$ & & \\
\hline \multicolumn{6}{|l|}{ Fracture classification } \\
\hline 31.A1 & $14(16.3)$ & $10(15.2)$ & $4(20.0)$ & Ref & \\
\hline $31 . A 2$ & $60(69.8)$ & $47(71.2)$ & $13(65.0)$ & $0.69(0.18-2.74)$ & 0.602 \\
\hline 31.A3 & $12(14.0)$ & $9(13.6)$ & $3(15.0)$ & $1.14(0.18-7.16)$ & 0.891 \\
\hline \multicolumn{6}{|l|}{ Fracture reduction quality } \\
\hline Satisfactory & $30(34.9)$ & $28(42.4)$ & $2(10.0)$ & Ref & \\
\hline Unsatisfactory & $56(65.1)$ & $38(57.6)$ & $18(90.0)$ & $10.1(1.31-77.6)$ & $0.027^{*}$ \\
\hline CalTAD (mm) & - & $27.3 \pm 6.4$ & $26.7 \pm 7.8$ & $0.96(0.89-1.05)$ & 0.358 \\
\hline TAD (mm) & - & $21.3 \pm 6.1$ & $22.4 \pm 6.4$ & $1.00(0.92-1.09)$ & 0.927 \\
\hline Cervical angle difference $\left({ }^{\circ}\right)$ & - & $-0.7 \pm 13.7$ & $-4.7 \pm 11.9$ & $0.98(0.94-1.03)$ & 0.481 \\
\hline AP Parker's ratio index & - & $52.6 \pm 8.0$ & $48.9 \pm 8.5$ & $0.95(0.89-1.01)$ & 0.102 \\
\hline Lateral Parker's ratio index & - & $47.2 \pm 8.2$ & $41.3 \pm 8.9$ & $0.95(0.89-1.00)$ & 0.062 \\
\hline
\end{tabular}

*A p-value < 0.05 was considered statistically significant. AP: anteroposterior; CalTaD: calcar referenced tip-apex distance; Cl: confidence interval, Ref: reference group; SD: standard deviation; TAD: tip-apex distance

satisfy the minimum of 90 days' radiological follow-up and were also excluded from the study. A further two patients were excluded as they underwent intramedullary nailing for nonosteoporotic pathological fractures. Of the remaining 370 patients who satisfied the inclusion criteria, $259(70.0 \%)$ were female and $111(30.0 \%)$ were male. 202 (54.6\%) had sustained a left-sided intertrochanteric fracture, while the remaining 168 (45.4\%) had sustained a right-sided fracture. The mean age at time of surgery of the included patients was 80.2 years.

Among the 370 fractures that were included in our study, $20(5.4 \%)$ cut-outs were identified. 16 of these were noted to be superior cut-outs and four were cut-throughs. All remaining non-cut-outs $(\mathrm{n}=350)$ were noted to have achieved radiographic union at final follow-up. The control group $(n=66)$ was defined by matching the cut-outs with the non-cut-outs by age at time of operation, gender and side of operation. There were no significant differences in age, side of operation or gender between the cut-out and control groups. The mean duration of radiological follow-up was 22 months in the cut-out group and 15 months in the control group. Of the 20 patients with cut-outs, seven eventually underwent revision to total hip replacement and were ambulant at final follow-up. The remaining 13 patients with cutouts were offered revision to total hip replacement but declined: one patient was treated with removal of implants after fracture varus malunion and 12 declined any further surgical intervention. All 13 patients with cut-outs who declined revision to total hip replacement developed radiographic features of osteoarthritis of the relevant hip joint and were noted to be wheelchair-bound at final follow-up.

Fracture reduction quality was assessed based on a modification of the method described by Baumgaertner et al. ${ }^{(7)}$ Within the control group, 28 (42.4\%) of the 66 fractures had satisfactory reduction, while $38(57.6 \%)$ had unsatisfactory reduction. Among the 20 patients in the cut-out group, $2(10.0 \%)$ had satisfactory fracture reduction, while $18(90.0 \%)$ had unsatisfactory reduction. Univariate analysis demonstrated that this difference was statistically significant (Table I). Fractures with unsatisfactory reduction quality were shown to be associated with a higher risk of cut-out as compared to those with satisfactory reduction quality $(p=0.027)$. The association between unsatisfactory fracture reduction quality and cut-outs remained significant on multivariate logistic regression analysis (OR 16.4, 95\% Cl 1.9-140.4, $\mathrm{p}=0.011$, not shown).

Fractures were classified based on the AO/OTA classification system, and fracture classification was not found to have a statistically significant association with cut-outs. Although the TAD in the cut-out group ( $22.4 \mathrm{~mm}$ ) was higher than in the control group $(21.3 \mathrm{~mm})$, this difference was not statistically significant. There were no statistically significant differences between the two groups with regard to CalTAD, AP Parker's ratio index, lateral Parker's ratio index or cervical angle difference.

Cut-outs were classified according to their pattern, namely cut-throughs or superior cut-outs, and analysed for differences between these two groups. Results of the subgroup analysis of cut-throughs and superior cut-outs are shown in Table II. Cutthroughs were associated with a significantly lower CalTAD (16.2 mm vs. $27.5 \mathrm{~mm} ; \mathrm{p}=0.016$ ) and lower AP Parker's ratio index (38.7 vs. 50.7; $p=0.007$ ) as compared to superior cut-outs. There was no significant difference in terms of age, gender, side of fracture, fracture classification, fracture reduction quality, TAD, cervical angle difference or lateral Parker's ratio index between the two groups.

\section{DISCUSSION}

The most common cause resulting in failure of fixation of intertrochanteric fractures is lag screw cut-out. ${ }^{(6,7,13)}$ However, on the whole, cut-outs are a fairly uncommon complication of intramedullary nailing of proximal femoral fractures. In our search of the current literature, the incidence of such cut-outs has been reported to be $0 \%-13 \% .^{(14-21)}$ At present, there is a relative scarcity of literature describing the predictors for cut-out with intramedullary fixation of intertrochanteric fractures. Our study 
Table II. Subgroup analysis of cut-throughs compared to superior cut-outs.

\begin{tabular}{|llll|}
\hline Parameter & \multicolumn{2}{c|}{ Median (interquartile range) } & p-value \\
\cline { 2 - 3 } & Cut-through $(\mathbf{n}=\mathbf{4})$ & Superior cut-out $(\mathbf{n}=\mathbf{1 6})$ & \\
\hline TAD $(\mathrm{mm})$ & $18.0(15.5$ to 25.0$)$ & $22.1(19.5$ to 29.3$)$ & 0.178 \\
\hline CalTAD $(\mathrm{mm})$ & $16.2(12.6$ to 24.2$)$ & $27.5(23.8$ to 35.5$)$ & $0.016^{*}$ \\
\hline Cervical angle difference $\left(^{\circ}\right)$ & $-5.6(-1.65$ to 18.8$)$ & $-3.9(-7.5$ to 1.9$)$ & 0.820 \\
\hline AP Parker's ratio index & $38.7(35.3$ to 43.8$)$ & $50.7(44.2$ to 58.4$)$ & $0.007^{*}$ \\
\hline Lateral Parker's ratio index & $44.9(31.5$ to 51.3$)$ & $40.9(34.4$ to 49.9$)$ & 0.554 \\
\hline
\end{tabular}

${ }^{*}$ A p-value < 0.05 was considered statistically significant. AP: anteroposterior; CalTAD: calcar referenced tip-apex distance; TAD: tip-apex distance

aims to address this void, especially as the use of intramedullary devices for fixation of intertrochanteric fractures is likely to continue to rise and that cut-out remains the primary mode of failure of such fixations.

To our knowledge, only four clinical studies ${ }^{(18-21)}$ have investigated predictors of cut-out based on samples that were purely treated with intramedullary devices. The earliest of these studies, by Geller et $\mathrm{al}^{\left({ }^{(18)}\right.}$ showed that a TAD > $25 \mathrm{~mm}$ was predictive of cut-out. A subsequent study by Lobo-Escolar et al ${ }^{(19)}$ found that higher TAD and suboptimal osteosynthesis were predictive. Kashigar et $\mathrm{al}^{(20)}$ reported that a higher CalTAD, a novel measurement index of implant position first described in a biomechanical study by Kuzyk et al, ${ }^{(12)}$ was predictive. Nikoloski et al,(21) in their study based on fixation with the PFNA, reported a bimodal distribution of cut-outs with an increased risk of cut-through or medial perforation with TAD $<20 \mathrm{~mm}$ and increased risk of superior cut-out with TAD $>30 \mathrm{~mm}$, leading the authors to conclude that the rule of TAD $<25 \mathrm{~mm}$ should not be applied to fixation with the PFNA. Instead, they recommended a TAD of 20-25 mm to reduce the incidence of both modes of cut-out.

These studies, however, were based on relatively small numbers of cut-outs: seven in Geller et al, ${ }^{(18)}$ ten in Kashigar et al ${ }^{(20)}$ and six in Nikoloski et $\mathrm{al}^{(21)}$, with the exception being Lobo-Escolar et al ${ }^{(19)}$ who reported 33 cut-outs. In all four studies, however, there was no standardisation in terms of the implants used, as each study had a variety of intramedullary implant brands as well as lengths. Furthermore, all four studies included subtrochanteric fractures in their study populations rather than exclusively studying intertrochanteric fractures.

To the best of our knowledge, we are the first centre to investigate the predictive factors for cut-out following proximal femoral nailing in a group consisting of purely intertrochanteric fractures using a standardised implant (200-mm Synthes PFNA). We found that $5.4 \%$ of patients undergoing intramedullary nailing of intertrochanteric fractures sustained cut-out of the helical blade.

Univariate analysis of our patients showed that unsatisfactory reduction quality was significantly associated with implant cutout. This was in line with the findings of Lobo-Escolar et al, ${ }^{(19)}$ who also reported an association between suboptimal osteosynthesis and the development of cut-outs in their study population. In that study, however, suboptimal osteosynthesis was defined by a less specific set of parameters that included lag screw positioning in the femoral head, diastasis of fracture fragments, varus or valgus neck-shaft angulation, and subjective assessment of fixation stability by two evaluating surgeons. The association of unsatisfactory reduction with cut-outs remained significant in our multivariate analysis. Our study, therefore, confirms the importance of ensuring satisfactory fracture reduction, as defined by the displacement and angulation criteria first described by Baumgaertner et $\mathrm{al},{ }^{(7)}$ in reducing the incidence of cut-outs in intramedullary nailing of intertrochanteric fractures.

In contrast to the findings of Geller et al ${ }^{(18)}$ Lobo-Escolar et al ${ }^{(19)}$ and Kashigar et al, ${ }^{(20)}$ our study did not show any association between TAD and cut-outs. TAD was $<25 \mathrm{~mm}$ in all but seven of our cut-outs and $>30 \mathrm{~mm}$ in only three cases, with the highest TAD among them being $32.4 \mathrm{~mm}$. This raises doubt about whether the rule of aiming for TAD $<25 \mathrm{~mm}$ as suggested by Geller et al ${ }^{(18)}$ should be applied to fixation with the 200-mm Synthes PFNA. There was also no association between cut-out and fracture classification, CalTAD, cervical angle difference, AP Parker's ratio index or lateral Parker's ratio index in our study.

Subgroup analysis of the cut-outs in our study showed that cut-throughs had significantly lower CaITAD and AP Parker's ratio index values than the superior cut-outs. Lower CaITAD and AP Parker's ratio values indicate that the cut-throughs had a more inferior and deeper position of the helical blade in the femoral head as compared to the superior cut-outs. This suggests the possibility of different mechanisms of cut-out in each of these two groups. Nikoloski et al, ${ }^{(21)}$ in their study investigating predictors of cut-out in proximal femoral fractures treated with the Synthes PFNA, also reported a similar bimodal distribution of cut-outs with four cases of superior cut-out and two cases of cut-through. Biomechanical studies comparing the helical blade design to the lag screw design have shown contrasting results, with studies by Sommers et $\mathrm{al}^{(22)}$ and Strauss et $\mathrm{al}^{(23)}$ showing superior cut-out resistance in helical blade designs, while Born et al $^{(24)}$ reported higher migration resistance in a hip screw design compared to the helical blade. The latter study, which compared the behaviour of various implants under cyclical loading to simulate the human gait cycle, showed that hip screws tended to migrate primarily in a cephalad direction, while helical blades exhibited distinct migration in an axial direction. Brunner et $\mathrm{al}^{(25)}$ reported three clinical cases of medial perforation of the helical blade through the femoral head, which the authors postulated may have been related to the helical blade design. Our study also demonstrates the phenomenon of cut-through with the helical blade design 
and suggests that it may be associated with a deeper and more inferior position of the helical blade in the femoral head. We therefore recommend a more central position of the helical blade in the AP view rather than an inferior position when using the PFNA or other similar intramedullary devices that employ the helical blade design.

The strength of our study lies in the fact that, to our knowledge, this is the first study investigating predictive factors for cut-out following proximal femoral nailing in a group consisting of purely intertrochanteric fractures using a standardised implant. Furthermore, our analysis was based on sample of 20 cases of cut-out, more than several other reports in the current literature.

The limitations of our study include its retrospective design, which restricted our ability to investigate other clinical parameters that may have influenced the development of implant cut-outs, such as medical comorbidities, postoperative weight-bearing status, severity of osteoporosis, if present, and premorbid functional level. Although the number of cut-outs examined in our study was greater than that in several other studies in the current literature, a sizeable number of patients were excluded due to inadequate radiological follow-up ( $<90$ days). This loss to follow-up is likely attributable to the study's older patient population, as older patients often fail to return due to a variety of reasons including transfer to a step-down rehabilitation facility or mortality secondary to their comorbid conditions. A prospective multicentre study would help to address these limitations and permit a more comprehensive investigation of clinical as well as radiological predictors of cut-outs.

In conclusion, the incidence of cut-outs among intertrochanteric fractures treated with the 200-mm PFNA was $5.4 \%$ in our study. Unsatisfactory fracture reduction quality was shown to be a significant predictor of cut-out in intertrochanteric fractures treated with the 200-mm Synthes PFNA. However, our study did not show any association between TAD and cut-out. Furthermore, we demonstrated significant differences between two distinct modes of cut-out, with cut-throughs being associated with a deeper and more inferior helical blade position in the femoral head compared to superior cut-outs. Based on these findings, we believe that the TAD $<25 \mathrm{~mm}$ guideline may not be applicable to the 200$\mathrm{mm}$ Synthes PFNA. We recommend that adequate attention be given to ensuring satisfactory fracture reduction to reduce the incidence of cut-outs during osteosynthesis of intertrochanteric fractures using the Synthes PFNA. We also recommend a central rather than inferior position of the helical blade in the femoral head to avoid possible cut-throughs. Prospective studies are needed to validate these findings and to investigate the influence of other factors such as medical comorbidities, osteoporosis and postoperative weight-bearing status on the development of implant cut-outs.

\section{REFERENCES}

1. Burge R, Dawson-Hughes B, Solomon DH, et al. Incidence and economic burden of osteoporosis-related fractures in the United States, 2005-2025. J Bone Miner Res 2007; 22:465-75.

2. Cummings SR, Rubin SM, Black D. The future of hip fractures in the United States. Numbers, costs, and potential effects of postmenopausal estrogen. Clin Orthop Relat Res 1990; (252):163-6.

3. Johnell O, Kanis JA. An estimate of the worldwide prevalence, mortality and disability associated with hip fracture. Osteoporos Int 2004; 15:897-902.

4. Autier $P$, Haentjens $P$, Bentin J, et al. Costs induced by hip fractures: a prospective controlled study in Belgium. Belgian Hip Fracture Study Group. Osteoporos Int 2000; 11:373-80

5. Braithwaite RS, Col NF, Wong JB. Estimating hip fracture morbidity, mortality and costs. J Am Geriatr Soc 2003; 51:364-70.

6. Davis TR, Sher JL, Horsman A, et al. Intertrochanteric femoral fractures. Mechanical failure after internal fixation. J Bone Joint Surg Br 1990; 72:26-31.

7. Baumgaertner MR, Curtin SL, Lindskog DM, Keggi JM. The value of the tip-apex distance in predicting failure of fixation of peritrochanteric fractures of the hip. J Bone Joint Surg Am 1995; 77:1058-64.

8. Haynes RC, Pöll RG, Miles AW, Weston RB. Failure of femoral head fixation: a cadaveric analysis of lag screw cut-out with the gamma locking nail and AO dynamic hip screw. Injury 1997; 28:337-41.

9. Parker MJ. Cutting-out of the dynamic hip screw related to its position. J Bone Joint Surg Br 1992; 74:625.

10. Pervez H, Parker MJ, Vowler S. Prediction of fixation failure after sliding hip screw fixation. Injury 2004; 35:994-8.

11. Baumgaertner MR, Solberg BD. Awareness of tip-apex distance reduces failure of fixation of trochanteric fractures of the hip. J Bone Joint Surg Br 1997; 79:969-71.

12. Kuzyk PR, Zdero R, Shah S, et al. Femoral head lag screw position for cephalomedullary nails: a biomechanical analysis. J Orthop Trauma 2012; 26:414-21

13. Bojan AJ, Beimel C, Speitling A, et al. 3066 consecutive gamma nails. 12 years experience at a single centre. BMC Musculoskelet Disord 2010; 11:133.

14. Mereddy P, Kamath S, Ramakrishnan M, Malik H, Donnachie N. The AO/ASIF proximal femoral nail antirotation (PFNA): a new design for the treatment of unstable proximal femoral fractures. Injury 2009; 40:428-32.

15. Pu JS, Liu L, Wang GL, Fang Y, Yang TF. Results of the proximal femoral nail anti-rotation (PFNA) in elderly Chinese patients. Int Orthop 2009; 33:1441-4.

16. Simmermacher RK, Ljungqvist J, Bail $\mathrm{H}$, et al; AO-PFNA study group. The new proximal femoral nail antirotation (PFNA) in daily practice: results of a multicentre clinical study. Injury 2008; 39:932-9.

17. Takigami I, Matsumoto K, Ohara A, et al. Treatment of trochanteric fractures with the PFNA (proximal femoral nail antirotation) nail system - report of early results. Bull NYU Hosp Jt Dis 2008; 66:276-9.

18. Geller JA, Saifi C, Morrison TA, Macaulay W. Tip-apex distance of intramedullary devices as a predictor of cut-out failure in the treatment of peritrochanteric elderly hip fractures. Int Orthop 2010; 34:719-22.

19. Lobo-Escolar A, Joven E, Iglesias D, Herrera A. Predictive factors for cutting-out in femoral intramedullary nailing. Injury 2010; 41:1312-6.

20. Kashigar A, Vincent A, Gunton MJ, et al. Predictors of failure for cephalomedullary nailing of proximal femoral fractures. Bone Joint J 2014; 96-B:1029-34.

21. Nikoloski AN, Osbrough AL, Yates PJ. Should the tip-apex distance (TAD) rule be modified for the proximal femoral nail antirotation (PFNA)? A retrospective study. J Orthop Surg Res 2013; 8:35.

22. Sommers $\mathrm{MB}$, Roth $\mathrm{C}$, Hall $\mathrm{H}$, et al. A laboratory model to evaluate cutout resistance of implants for pertrochanteric fracture fixation. J Orthop Trauma 2004; 18:361-8.

23. Strauss E, Frank J, Lee J, Kummer FJ, Tejwani N. Helical blade versus sliding hip screw for treatment of unstable intertrochanteric hip fractures: a biomechanical evaluation. Injury 2006; 37:984-9.

24. Born C, Karich B, Bauer C, von Oldenburg G, Augat P. Hip screw migration testing: first results for hip screws and helical blades utilizing a new oscillating test method. J Orthop Res 2011; 29:760-6.

25. Brunner A, Jöckel JA, Babst R. The PFNA proximal femur nail in treatment of unstable proximal femur fractures--3 cases of postoperative perforation of the helical blade into the hip joint. J Orthop Trauma 2008; 22:731-6. 